\title{
PERFIL METABÓLICO DE AVES COMERCIALES MEDIANTE MÉTODOS DIRECTOS
}

\author{
Metabolic Profile of Commercial Birds Using Direct Methods
}

Jancy Darly Flórez ${ }^{1,3}$, José Henry Osorio ${ }^{2,4}$

\section{Resumen}

En el presente estudio se analizó y comparó los niveles de colesterol de las lipoproteínas de baja y alta densidad en pollos de engorde y gallinas ponedoras, para determinar la lipoproteína que se encuentra en mayor concentración, empleando métodos de análisis que no se afectan por las altas concentraciones de triglicéridos. Se tomaron muestras en ayunas a 30 pollos de engorde de la línea Cobb 500 de 35 días de edad y a 40 gallinas ponedoras de la línea Hy Line W-36 con 26 semanas de edad. Se cuantificó los niveles del colesterol de las lipoproteínas de baja (LDL) y alta (HDL) densidad por los métodos directos a base de detergentes y N,N-bis(4-sulfobutil)-m-toluidina. El nivel de lipoproteínas de HDL fue mayor, tanto en los pollos de engorde (93.1 \pm 16 vs. $52.2 \pm 9.1 \mathrm{mg} / \mathrm{dL})$ como en las gallinas ponedoras $(61.7 \pm 15.4$ vs. $49.1 \pm 12.6 \mathrm{mg} / \mathrm{dL})$. Se concluye que utilizando los métodos directos a base de detergentes y N,N-bis(4-sulfobutil)-m-toluidina, las aves comerciales tienen como patrón metabólico de lípidos a las lipoproteínas de alta densidad.

Palabras clave: colesterol HDL, colesterol LDL, gallinas ponedoras, pollos de engorde

\section{Abstract}

This study measured and compared the levels of low and high-density lipoprotein cholesterol in broilers and laying hens to determine the type of lipoprotein in higher concentration, using an analytical method that cannot be affected by high concentrations of triglycerides. Blood samples were collected in 30 broilers (Cobb 500) of 35-day-old and in 40 laying hens (Hy-Line W-36) 26 weeks old. Cholesterol levels of low and high density lipoproteins were measured by direct methods based on detergents and N,N-Bis(4-sulfobutyl)-m-toluidine. The

\footnotetext{
${ }^{1}$ Departamento de Salud Animal, ${ }^{2}$ Laboratorio de Bioquímica Clínica y Patología Molecular, Departamento de Ciencias Básicas de la Salud, Universidad de Caldas, Manizales, Colombia

${ }^{3}$ E-mail: jancydarly@yahoo.com.ar

${ }^{4}$ E-mail:jose.osorio_o@ucaldas.edu.co
} 
results showed that high density lipoprotein cholesterol was more abundant in both broilers (93.1 \pm 16 vs. $52.2 \pm 9.1 \mathrm{mg} / \mathrm{dL})$ and laying hens $(61.7 \pm 15.4$ vs. $49.1 \pm 12.6 \mathrm{mg} / \mathrm{dL})$. It was concluded that using direct methods based on detergents and N,N-Bis (4sulfobutyl)-m-toluidine, the high density lipoprotein is the metabolic pattern of lipids in commercial poultry.

Key words: HDL cholesterol, LDL cholesterol, laying hens, broilers

\section{INTRODUCCIÓN}

En los mamíferos es común encontrar que las lipoproteínas de baja densidad (LDL, del inglés low density lipoprotein) que realizan el transporte directo del colesterol o las lipoproteínas de alta densidad (HDL, del inglés high density lipoprotein) que realizan el transporte inverso del colesterol, son más abundantes las unas que las otras, según sea la especie animal sin importar el sexo, y a esto se le conoce como patrón metabólico de los lípidos.

En las aves comerciales (Gallus domesticus) ocurre lo contrario, ya que el perfil lipídico difiere entre sexos (Hermier et al., 1989). En el pollo se ha encontrado que las HDL son más abundantes que las LDL (Chen et al., 2005; Velasco et al., 2010); además, el nivel de triglicéridos es de aproximadamente $42 \mathrm{mg} / \mathrm{dL}$ (Chen et al., 2005), aunque hay estudios que demuestran lo contrario (Musa et al., 2006); no obstante, estas diferencias podrían deberse al método de análisis utilizado en la cuantificación de las lipoproteínas.

En el caso de las gallinas ponedoras en producción el comportamiento es diferente, ya que por acción de los estrógenos se altera el metabolismo de las lipoproteínas, aumentando significativamente las lipoproteínas de muy baja densidad, las cuales transportan los triglicéridos que van a depositarse en los oocitos en crecimiento (Walzem et al., 1999), llegando estos hasta niveles de $1200 \mathrm{mg} / \mathrm{dL}$
(Yue et al., 2011); y a su vez, los estrógenos disminuyen las lipoproteínas de alta densidad (Hermier et al., 1989). Por esta razón, se indica que las lipoproteínas de baja densidad son más abundantes en las gallinas cuando no se tienen en cuenta los niveles de lipoproteínas de muy baja densidad (VLDL, del inglés very low density lipoprotein).

Los métodos de evaluación del perfil lipídico en las aves comerciales son los mismos que se utilizan en los humanos, y entre estos están la fórmula de Friedewald (Musa et al., 2006; Salma et al., 2007) y los métodos de precipitados o directos (Yue et al., 2011). En algunos casos se ha empleado el método de ultracentrifugación (Hermier et al., 1984, 1989) a pesar de ser un método poco práctico y costoso (Palacios et al., 1999). No obstante, con el uso de estos métodos enzimáticos-colorimétricos se puede incurrir en errores, especialmente en las gallinas ponedoras, pues los altos niveles de triglicéridos $(>400 \mathrm{mg} / \mathrm{dL})$ afectan la medición de las HDL y las LDL, especialmente con la fórmula de Friedewald (Friedewald $e t$ al., 1972) y con los métodos de precipitado (Demacker et al., 1980; Nauck et al., 2002). Por otro lado, los métodos directos se caracterizan por estar libres de alteraciones endógenas, tales como los causados por altos niveles de triglicéridos sanguíneos (Nauck et al., 2002).

En el presente trabajo se cuantificó y comparó los niveles de colesterol HDL y LDL en pollos de la línea Cobb 500 y en gallinas de la línea Hy-Line W-36, y se identificó la 
lipoproteína más abundante sin tener en cuenta las VLDLs, utilizando los métodos directos a base de detergentes y N,N-bis(4sulfobutil)-m-toluidina (DSBmT), los cuales son recomendados en humanos con niveles de triglicéridos superiores a $400 \mathrm{mg} / \mathrm{dL}$.

\section{Materiales y Métodos}

\section{Manejo de los Pollos de Engorde}

Se emplearon 30 pollos de la línea Cobb 500 de 21 días de edad, de una granja de la empresa Zarpollo, ubicada en Chinchiná, Caldas, Colombia. Las aves se mantuvieron en un cuarto de ambiente controlado a una temperatura promedio de $25^{\circ} \mathrm{C}$ y con 12 horas de luz. Fueron alimentados durante 15 días con una dieta a base de maíz y torta de soya (Cuadro 1).

\section{Manejo de las Gallinas Ponedoras}

Se emplearon 40 gallinas de la línea HyLine W-36, criadas en la Granja Montelindo de la Universidad de Caldas, a una temperatura promedio de $25{ }^{\circ} \mathrm{C}$ y con 13 horas de luz. Las aves fueron elegidas al azar y alimentadas hasta la semana 24 de edad con una dieta comercial para gallinas ponedoras, junto con todo el lote de aves; sin embargo, en las semanas 25 y 26 de edad fueron alimentadas con una dieta a base de maíz y torta de soya (Cuadro 1).

\section{Recolección y Análisis de las Muestras}

Se realizó un ayuno de $12 \mathrm{~h}$ previo a la toma de la muestra. Se colectó $20 \mathrm{ml}$ de sangre directamente de la yugular de todas las aves. La sangre se centrifugó a $3000 \mathrm{rpm}$ durante 15 minutos (Thermo Scientific IEC CL40R Refrigerated Benchtop Centrifuge, 1210927, Alemania) y el suero resultante se congeló a -30 ${ }^{\circ} \mathrm{C}$ (Revco ULT350-5-D12, Asheville, EEUU) hasta su procesamiento. $\mathrm{Al}$ momento de procesar las muestras, los sueros fueron descongelados a temperatura ambiente, y luego se llevaron al baño maría a una temperatura de $37^{\circ} \mathrm{C}$ por 10 minutos.

Los valores colesterol HDL y LDL se determinaron por métodos enzimáticoscolorimétricos. Los reactivos para la determinación del colesterol HDL y LDL mediante los métodos directos se basan en detergentes y DSBmT. El análisis se hizo según las recomendaciones del fabricante (Laboratorios BioSystems, España) empleando un espectrofotómetro Rayto RT-1904C (Rayto Life and Analytical Sciences, China).

\section{Análisis Estadístico}

Los datos fueron analizados mediante un análisis de varianza simple, empleando el paquete estadístico Statgraphics Plus 5.1 (Statistical Graphics Corporation. Statgraphics Plus, v. 5.1).

\section{Resultados}

Las medias de los valores del colesterol de las lipoproteínas de alta y baja densidad en pollos de engorde y gallinas ponedoras se muestran en el Cuadro 2. En todos los pollos, los valores de colesterol HDL fueron mayores en todas las aves $(\mathrm{p}<0.05)$, en tanto que en 37 de las 40 gallinas el colesterol HDL fue mayor al colesterol LDL $(\mathrm{p}<0.05)$.

\section{Discusión}

Los resultados coinciden con trabajos realizados en aves comerciales, donde se ha demostrado que las HDL dominan sobre la LDL en los pollos de engorde, pero sin que exista un efecto de raza (Musa et al., 2007), castración (Chen et al., 2005) o dieta (Cao et al., 2012; Velasco et al., 2010). Sin embargo, Musa et al. (2006) encontraron niveles de LDL superiores a los valores de HDL en pollos de tipo magro, utilizando la fórmula de Friedewald, lo cual podría ser la causa de 
Cuadro 1. Composición de la dieta experimental para pollos de engorde de la línea Cobb 500 de 21 días de edad y gallinas ponedoras

\begin{tabular}{lcc}
\hline & Pollos de engorde & Gallina ponedora \\
\hline Materia seca & 93.48 & 93.05 \\
Nitrógeno total & 3.02 & 3.60 \\
Proteína bruta & 18.88 & 17.50 \\
Grasa total & 2.10 & 2.32 \\
Fibra bruta & 4.09 & 3.04 \\
Cenizas & 6.41 & 6.41 \\
Fósforo & 0.45 & 0.22 \\
Calcio & 0.70 & 0.46 \\
\hline
\end{tabular}

Cuadro 2. Comparación entre los valores de colesterol HDL y LDL (promedio \pm desviación estándar) en pollos de engorde y gallinas ponedoras

\begin{tabular}{lcc}
\hline & C-HDL $^{1}$ & C-LDL $^{2}$ \\
\hline Pollos de engorde (Cobb 500) & $93.1 \pm 16.0^{\mathrm{a}}$ & $52.2 \pm 9.1^{\mathrm{b}}$ \\
Gallinas ponedoras (Hy-Line W-36) & $61.7 \pm 15.4^{\mathrm{a}}$ & $49.1 \pm 12.6^{\mathrm{b}}$ \\
\hline${ }^{1}$ Colesterol de la lipoproteína de alta densidad & & \\
2Colesterol de la lipoproteína de baja densidad \\
${ }^{\mathrm{a}, \mathrm{b}}$ Superíndices diferentes dentro de líneas indican diferencia estadística $(\mathrm{p}<0.05)$
\end{tabular}

esta diferencia con el presente estudio y con los otros trabajos publicados. Se ha comprobado que este procedimiento no debe ser empleado en pollos de engorde por la dependencia que tiene de los niveles de triglicéridos, colesterol total y colesterol HDL para dar los resultados (Flórez, 2012).

El caso de las tres gallinas con mayores niveles de LDL en relación a los niveles de HDL pudo deberse a que los niveles de estrógenos en las gallinas no solo disminuyen los niveles de HDL, sino que también alteran la composición de todas las lipoproteínas plasmáticas. Esto, aunado a los inconvenientes del método de ultracentrifugación
(Hermier et al., 1989) que dificultan la lectura pudiendo afectar la respuesta con el método evaluado.

En trabajos donde han evaluado el perfil lipídico en gallinas ponedoras con el método de ultracentrifugación en combinación de kits enzimáticos para colesterol, se ha encontrado que el tipo de dieta puede afectar las concentraciones de colesterol HDL y colesterol LDL (An et al., 1997). Sin embargo, en un experimento donde no solo se cambió la dieta sino que, además, se evaluó el comportamiento de dos líneas de ponedoras con los niveles de ácido linoleico conjugado de esta, se pudo demostrar que los niveles de colesterol HDL fueron siempre superiores a 
los del colesterol LDL. Por otro lado, en un estudio donde se evaluaron bacterias del género Rhodobacter en la dieta de gallinas ponedoras (Salma et al., 2007), utilizando un método de precipitado para la cuantificación del colesterol HDL y la fórmula de Friedewald para el colesterol LDL, se mostraron niveles elevados de colesterol LDL en comparación con los niveles de colesterol HDL.

\section{Conclusiones}

Las aves comerciales, tanto pollos de engorde como gallinas ponedoras, tienen como patrón metabólico de lípidos a las lipoproteínas de alta densidad (HDL).

\section{Literatura Citada}

1. An BK, Nishiyama H, Tanaka K, Ohtani S, Iwata T, Tsutsumi K, Kasai $M .1997$. Dietary safflower phospholipid reduces liver lipids in laying hens. Poultry Sci 76: 689-695.

2. Cao FL, Zhang XH, Yu WW, Zhao LG, Wang T. 2012. Effect of feeding fermented Ginkgo biloba leaves on growth performance, meat quality, and lipid metabolism in broilers. Poultry Sci 91: 1210-121.

3. Chen KL, Chi WT, Chiou PWS. 2005. Caponization and testosterone implantation effects on blood lipid and lipoprotein profile in male chickens. Poultry Sci 84: 547-552.

4. Demacker PNM, Vos-Janssen HE, Humans AGM, Laar Avt, Jansen P. 1980. Measurement of high-density lipoprotein cholesterol in serum: comparison of six isolation methods combined with enzymic cholesterol analysis. Clin Chem 26: 1780-1786.

5. Flórez JD. 2012. Estudio básico del metabolismo lipídico en aves comerciales. Tesis de Maestría. Manizales, Colombia: Universidad de Caldas. 89 p.
6. Friedewald W, Levy $R$, Fredrickson D. 1972. Estimation of the concentration of low-density lipoprotein cholesterolin plasma, without use of the preparative ultracentrifuge. Clin Chem 18: 499-502.

7. Hermier D, Chapman J, Leclercq B. 1984. Plasma lipoprotein profile in fasted and refed chickens of two strains selected for high or low adiposity. J Nutr 114: 1112-1121.

8. Hermier D, Forgez P, Williams J, Chapman MJ. 1989. Alterations in plasma lipoproteins and apolipoproteins associated with estrogen-induced hyperlipidemia in the laying hen. Eur $\mathbf{J}$ Biochem 184: 109-118.

9. Musa HH, Chen GH, Cheng JH, Yousif GM. 2007. Relation between abdominal fat and serum cholesterol, triglycerides, and lipoprotein concentration in chicken breeds. Turk $\mathbf{J}$ Vet Anim Sci 31: 375-379.

10. Musa HH, Chen GH, Wang KH, Li BC, Mekki DM, Shu JT, Ju HP. 2006. Relation between serum cholesterol level, lipoprotein concentration and carcass characteristics in genetically lean and fat chicken breeds. J Biol Sci 6: 616620.

11. Nauck M, Russell WG, Rifai N. 2002. Methods for measurement of LDLcholesterol: A critical assessment of direct measurement by homogeneous assays versus calculation. Clin Chem 48: 236-254.

12. Palacios M, Esteban M, Aguila JA, Ortolá J. 1999. Recomendaciones para la determinación de la concentración en suero de colesterol de las lipoproteínas de alta densidad. Quim Clín 18: 33-40.

13. Salma U, Miah AG, Tareq KMA, Maki T, Tsujii H. 2007. Effect of dietary Rhodobacter capsulatus on egg-yolk cholesterol and laying hen performance. Poultry Sci 86: 714-719.

14. Velasco S, Ortiz LT, Alzueta C, Rebole A, Trevino J, Rodriguez ML. 2010. Effect of inulin supplementation and dietary fat source on performance, blood serum metabolites, liver lipids, abdominal fat deposition, and tissue fatty acid 
composition in broiler chickens. Poultry Sci 89: 1651-1662.

15. Walzem RL, Hansen RJ, Williams DL, Hamilton RL. 1999. Estrogen induction of VLDL assembly in egg-laying hens. J Nutr 129: 467S-472S.
16. Yue HY, Wang J, Qi XL, Ji F, Liu MF, Wu SG, Zhang HJ, Qi GH. 2011. Effects of dietary oxidized oil on laying performance, lipid metabolism, and apolipoprotein gene expression in laying hens. Poultry Sci 90: 1728-1736. 